\title{
Practical development of efficient thermoelectric - Photovoltaic hybrid systems based on wide-gap solar cells
}

\author{
Bruno Lorenzi $^{\text {a, *, Paolo Mariani }}{ }^{\mathrm{b}}$, Andrea Reale ${ }^{\mathrm{b}}$, Aldo Di Carlo ${ }^{\mathrm{b}, \mathrm{c}}$, Gang Chen ${ }^{\mathrm{d}}$, \\ Dario Narducci ${ }^{\text {a }}$
}

${ }^{\text {a }}$ Dept. Materials Science, Univ. of Milano Bicocca, Milano 20125, Italy

b CHOSE, Center for Hybrid and Organic Solar Energy, University of Rome Tor Vergata, Rome 00133, Italy

${ }^{\mathrm{c}}$ ISM-CNR, Institute of Structure of Matter, National Research Council, Rome 00133, Italy

d Dept. Mechanical Engineering, Massachusetts Institute of Technology, Cambridge, MA 02139, USA

\section{H I G H L I G H T S}

- The efficiency gain due to hybridization of wide-gap PV was theoretically evaluated.

- The optimized working temperatures and thermoelectric generator layout were found.

- A hybrid device based on a perovskite solar cell and a $\mathrm{Bi}_{2} \mathrm{Te}_{3}$ TEG was developed.

\section{A R T I C L E I N F O}

\section{Keywords:}

Hybrid

Photovoltaic

Thermoelectric

\begin{abstract}
A B S T R A C T
The decrease of solar cell efficiency with temperature is a known problem for photovoltaics (PV). Temperature sensitivity can lead to a considerable amount of energy losses over the lifetime of solar panels. In this perspective Hybrid Thermoelectric-Photovoltaic (HTEPV) systems, which recover solar cell heat losses to produce an additional power output, can be a suitable option. However only hybridization of wide-gap solar cells is convenient in terms of efficiency gains and deserves investigation to evaluate HTEPV devices effectiveness. In this work we report the modeling and the development of customized bismuth telluride thermoelectric generators, optimized to be hybridized with amorphous silicon (aSi), Gallium Indium Phosphide (GaInP) or Perovskites solar cells. The model results showed in all three cases efficiency gains with a maximum of $+3.1 \%$ for Perovskites (from $16.4 \%$ to $19.5 \%$ ). These enhancements were then experimentally validated for the case of Perovskites solar cells, for which maximum gains were found to occur at typical operating temperatures of conventional PVs. This experimental evaluation demonstrated in an accurate fashion the real potential of thermoelectric hybridization of solar cells.
\end{abstract}

\section{Introduction}

Silicon solar cells dominate the photovoltaic (PV) market. Their high efficiencies, along with their relatively low cost, make them the most valuable solution nowadays. However, it is well known that silicon solar cells are extremely sensitive to temperature, and that they can lose up to $15-20 \%$ of their room temperature efficiency under normal operating conditions $[1,2]$. Wide-gap solar cells have been proposed as valuable alternative solutions, because to their smaller temperature sensitivity [3]. However, the room temperature efficiencies of these candidate materials are still lower than silicon-based devices, making them not competitive enough. The reasons for these lower performances is a smaller readiness level of the materials and of the devices based on widegap absorbers [4].

A suitable option to enhance the competitiveness of wide-gap solar cells more rapidly is to implement heat recovery strategies. It is well know that for single-junction solar cells the efficiency are limited by the so-called Shockley-Quisser limit [5], and that most of the losses in solar cells (up to 90\%) are in the form of heat [6-10]. Among all the suitable options in order to enhance PV efficiencies by heat recovery, the hybridization with thermoelectric generators (TEGs), has gained an increasing attention in recent years. In Hybrid Thermoelectric-

\footnotetext{
* Corresponding author.

E-mail address: bruno.lorenzi@unimib.it (B. Lorenzi).
} 
Photovoltaic (HTEPV) systems, the TEG part can recover the solar cell heat to produce an additional power output and enhance the overall device output power and efficiency. HTEPV devices have been the object of many studies and reviews, especially in the last years [11-13]. Within this literature the opinion on the actual effectiveness of HTEPV devices has been quite fluctuating. Actually along the years these systems have been reported as both very convenient [14-17] and not suitable to increase PV efficiency $[18,19]$. In the last couple of years most of the works published on HTEPV systems have been dedicated to the study of their transient behavior over time [20-22], to the effect of the TEG geometrical parameters [23,24], and to hybrids with perovskites solar cells $[25,26]$. In particular for perovskites, Zhou et al. [25] reported a surprising $4.9 \%$ efficiency gain for a $\mathrm{CH}_{3} \mathrm{NH}_{3} \mathrm{~Pb}\left(\mathrm{I}_{0.95} \mathrm{Br}_{0.05}\right)_{3}$ solar cell with optimized energy gap, coupled with a commercial TEG. Strangely enough the authors report a $\Delta \mathrm{T}$ over the TEG sides of only $7.7^{\circ} \mathrm{C}$ corresponding to the mentioned efficiency gain, making the interpretation of this result quite difficult. A possible explanation can be related to the different renormalization of the power densities over the respective areas of the two parts of the hybrid device. Fu et al. [26] reported instead the hybridization of a large area perovskite solar module with a customized TEG based on bismuth telluride. In this case, the authors showed a more sensible $1 \%$ efficiency gain reaching a total hybrid efficiency of $12.7 \%$. The work is particularly interesting for the study of the electrical hybridization of the two system parts, and for the analysis of the efficiency stability over time.

These preliminary results on the thermoelectric hybridization with perovskite solar cells, call for further analysis of the potential of HTEPV systems with this kind of material, and in general with wide-gap solar cells. This is the approach we follow in this work. In fact here we present a theoretical and experimental study on HTEPV systems based on widegap solar cells. Based on a detailed characterization of three kinds of wide-gap cells, namely amorphous silicon (aSi), Gallium Indium Phosphide (GaInP) and Perovskites solar cells, we exploited a theoretical model to predict the hybrid system behavior versus temperature. The model results were then used to choose the best PV material for the thermoelectric hybridization (namely the perovskite solar cell), and to define the TEG design. An optimized bismuth telluride TEG was then developed and characterized on the basis of previous studies on pure Solar Thermoelectric Generators (STEGs) [27-29]. Finally, the HTEPV devices were built and characterized as a function of temperature, optical concentration, ambient pressure, and cold side cooling. This experimental evaluation validated the theoretical model used in this work and demonstrated the actual potential of HTEPV system based on wide-gap solar cells.

Our approach is novel in many respects. First of all, in this work a home-made TEG is developed to reach thermal matching conditions making the system work at the optimal temperature. In fact, very rarely commercial TEGs have a thermal resistance suitable for solar cells hybridization, as pointed out in our previous work [30]. The optimized layout was found based on a deep characterization of the solar cell to be hybridized. Furthermore, we focused our efforts on a very specific target, namely the demonstration of an effective HTEPV operating at low optical concentrations and low temperatures. To this purpose only wide-gap solar cells are suitable because they are less sensitive to temperature. In this perspective, this work not only confutes the thesis that only high optical concentrations are suitable for HTEPV systems [18], but also identifies a class of already existing implementable materials. Finally, the study on the effect of the vacuum level and of the cold side temperature add a perspective on the applicability of this kind of HTEPV systems in real-world scenarios. Therefore, the main objective of this work is the development and the characterization of a HTEPV prototype optimized to work at low optical concentration, demonstrating efficiency gains compared to the sole PV case. The methodology adopted starts from a deep characterization of the solar cell to be implemented, and the use of a theoretical model to predict the optimized hybrid design. Based on this model a home-made TEG, based on Bi2Te3, is constructed and paired with the solar cells for characterization.

\section{System description and modeling}

The hybrid system developed in this work consists of an optimized TEG placed in thermal contact with the back of a wide-gap solar cells, as depicted in Fig. 1. In this kind of devices the solar cell absorbs sunlight and produces an electrical power which is collected at its front and back contacts. Most of the not converted power becomes heat within the solar cell and increases its temperature. The TEG (in this case composed by only one couple made of a p-type and a n-type leg) absorbs part of this heat and produces and additional electrical power by means of the Seebeck effect. This kind of thermally coupled generators should not be confused with another class of HTEPV systems, normally referred as optically coupled devices [31,32]. In that case the TEG and PV parts are in fact separated, and a spectrum splitting technology is implemented to direct different parts of the solar spectrum towards them. In a previous study we showed how the optically coupled approach is less convenient than the thermally coupled one [33]. When the TEG is not in thermal contact with the solar cell, it can only access the infrared (IR) part of the solar spectrum, without being able to recover heat losses of the PV part. Most of the heat losses are in fact happening in the UV-Vis section of the spectrum where the solar cells absorb [9]. In other words, it is more convenient, in terms of final efficiency gains, to keep the solar cell at the same temperature of the TEG hot side, instead of keeping the cell cold but loosing much of the recoverable heat. For this reason, the temperature sensitivity of the PV part is a key parameter, and the kind of solar cells to be hybridized must be chosen carefully. Temperature sensitive materials, such as silicon, loose too much efficiency to make the hybridization convenient. On the other hand, wide-gap solar cells are less temperature sensitive so that their thermoelectric hybridization might be convenient, as shown in our previous work [34]. The physical reason behind this behavior relies on the fact that temperature sensitivity depends on the electron-hole recombination rate. The wider the energy gap, the smaller the recombination rate, and consequently the temperature sensitivity. For a detailed discussion on this matter the reader may refer to [35].

Another consideration deals with the electrical connection between the PV and TEG parts. The case in which the two components are electrically separated is the simpler. For this kind of systems the thermal flux flowing through the device is the main parameter on which designing the HTEPV components. For the case of electrical hybridization, instead, also the internal resistance of the PV and TEG parts must be considered, and a current matching optimization must be considered. Several works have been devoted to electrically hybridized systems with the conclusion that in general it is possible to match the two components without having additional losses $[16,36]$. In this perspective, the case in which the PV and TEG parts are separated is a simplification of the fully hybridized case and it is the one implemented in this paper.

Summarizing, in this work we developed a thermally coupled, electrically separated, HTEPV device based on wide-gap solar cells. The working mechanism can be outlined as follows. The solar cell absorbs sunlight and converts it into electrical power. The unconverted part mostly becomes heat in the cell. Part of this heat then flows through the TEG, which is placed in thermal contact with the cell back at its hot plate and is cooled down by a fluid dissipater at its cold plate. The TEG converts part of the heat flowing through it into an additional electrical output. The dimensions of the TEG legs are designed to increase as much as possible the overall efficiency of the system. Finally, the hybrid device is placed into an evacuated tube, in order to minimize the heat exchange with the environment.

For such kind of systems the hybrid efficiency can be written as

$\eta_{\text {htepv }}=\eta_{p v}+\eta_{\text {teg }} \eta_{o t}$

where $\eta_{p v}$ and $\eta_{\text {teg }}$ are respectively the temperature dependent PV and 

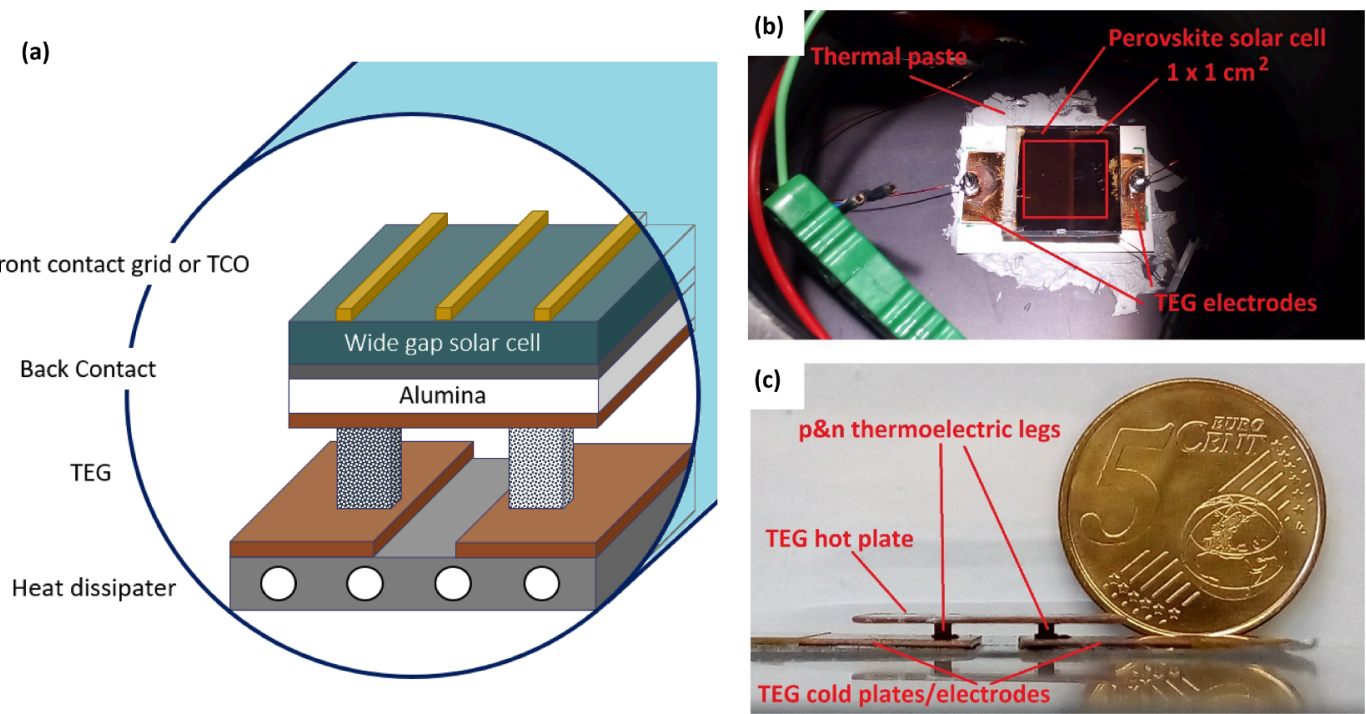

Fig. 1. The HTEPV system object of this work. (a) Scheme of the device, with the various parts composing the system. (b) Picture of the hybrid device placed in the vacuum chamber, viewed from the top. The solar cell active area is $1 \mathrm{~cm}^{2}$ as show by the red square (a metallic mask was used during characterization in order to cover the remain part). (c) Side view of the optimized TEG. (For interpretation of the references to colour in this figure legend, the reader is referred to the web version of this article.)

TEG efficiencies, and $\eta_{o t}$ the so-called opto-thermal efficiency.

The PV efficiency is normally reported to have a linear decrease as a function of temperature [37]. In this work $\eta_{p v}$ is determined by a detailed characterization of the wide-gap solar cells chosen. The TEG efficiency $\left(\eta_{\text {teg }}\right)$ is instead calculated on the material thermoelectric properties as follows [38]

$\eta_{\text {teg }}=\frac{T_{h}-T_{c}}{T_{h}} \frac{\sqrt{1+Z_{p n} T_{m}}-1}{\sqrt{1+Z_{p n} T_{m}}+\frac{T_{c}}{T_{h}}}$

with $T_{h}$ and $T_{c}$ respectively the hot and cold temperatures of the TEG, $T_{m}=\frac{T_{h}+T_{c}}{2}$, and $Z_{p n}$ the thermoelectric figure of merit defined as

$Z_{p n}=\frac{\left(\alpha_{p}-\alpha_{n}\right)^{2}}{\left(\sqrt{\kappa_{p} \rho_{p}}+\sqrt{\kappa_{n} \rho_{n}}\right)^{2}}$

where $\alpha, \rho$, and $\kappa$ are the Seebeck coefficient, the electrical resistivity, and the thermal conductivity of the $\mathrm{p}$ and $\mathrm{n}$ thermoelectric materials. In this work the TEG was developed starting from commercial bismuth telluride $\left(\mathrm{Bi}_{2} \mathrm{Te}_{3}\right)$ wafers, and the thermoelectric properties were given by the producer. Once the TEG was built its electrical properties were then characterized and compared with the producer's data.

The opto-thermal component $\eta_{o t}$ of Eq. (1) is instead the efficiency with which the HTEPV system converts the incoming power into heat flowing through the thermoelectric part. Actually, not all the solar power is converted into heat flowing through the TEG, as some of it is reflected by the solar cell top surface, and part of the heat generated within the solar cell is lost towards the environment. In the present work we consider placing the HTEPV device encapsulated within an evacuated environment. Thus, only radiative losses have to be taken into account. In this perspective the opto-thermal efficiency can be written as [39]

$\eta_{o t}=\left[\left(1-\eta_{p v}\right)\left(1-R_{p v}\right) \tau_{e n c}\right]-\frac{Q_{\text {rad }}}{P_{\text {in }}}$

with $R_{p v}$ and $\tau_{e n c}$ respectively the solar cell optical reflectance and the encapsulation optical transmittance, and with $P_{\text {in }}$ and $Q_{\text {rad }}$ respectively the solar input power and the sum of all the radiative heat losses. In Eq. (4) the product of the terms in the square brackets is the efficiency with which the solar cell converts the input solar power into heat. We assume therefore that all the power not becoming electrical output within the solar cell, excluding optical losses (namely $R_{p v}$ and $\tau_{\text {enc }}$ ) becomes heat. This assumption is justified on the basis of estimations of heat losses available in solar cells [9].

The input power can be written instead as

$P_{\text {in }}=\gamma_{\text {opt }} G A_{a b s}$

where $\gamma_{\text {opt }}, G$, and $A_{a b s}$ are respectively the optical concentration, the input solar power density, and the solar cell active area.

Heat losses $\left(Q_{\mathrm{rad}}\right)$ account for radiative losses between the solar cell top surface and the environment, and between the TEG hot and cold sides. Following the model of our previous study [39], it can be shown that

$Q_{\text {rad }}=2 \varepsilon_{\text {eff }} \sigma A_{a b s}\left(T_{h}^{4}-T_{c}^{4}\right)$

where $\sigma$ is the Stefan-Boltzmann constant and $\varepsilon_{\text {eff }}$ is an effective emittance accounting for both sources of radiative loss. Eq. (6) is a simplification that can be made by taking some assumptions. Actually, the radiative losses from the PV top surface should be written as

$Q_{\text {rad }}^{p v}=\varepsilon_{p v} \sigma A_{a b s}\left(T_{p v}^{4}-T_{a}^{4}\right)$

where $\varepsilon_{p v}$ is the top surface solar cell emittance, $T_{p v}$ the PV temperature, and $T_{a}$ the environment temperature; while the radiative loss across the TEG is

$Q_{\text {rad }}^{\text {teg }}=\varepsilon_{\text {teg }} \sigma\left(A_{\text {teg }}-A_{\text {te }}\right)\left(T_{h}^{4}-T_{c}^{4}\right)$

where $A_{t e g}$ is TEG area, $A_{t e}$ is the area occupied by thermoelectric legs, and $\varepsilon_{\text {teg }}$ is the effective TEG emittance accounting for radiative exchange from the hot to the cold plates, and from the cold to the hot plates of the TEG. It can be shown that [40]

$\varepsilon_{\text {teg }}=\frac{1}{\frac{1}{\varepsilon_{h}}+\frac{1}{\varepsilon_{c}}-1}$

with $\varepsilon_{h}$ and $\varepsilon_{c}$ respectively the emittances of the hot and cold TEG plates.

Eqs. (7) and (8) can be joined to give Eq. (6), if

1. the PV and TEG parts have the same areas $\left(A_{a b s}=A_{\text {teg }}\right)$ 
2. the total TEG area is much bigger than the area occupied by thermoelectric legs $\left(A_{t e g} \gg A_{t e}\right)$

3. the PV have the same temperature of the TEG hot side $\left(T_{p v}=T_{h}\right)$

4. the TEG cold side is at room temperature $\left(T_{c}=T_{a}\right)$

If all these assumptions are satisfied, it follows that $A_{a b s}=A_{\text {teg }}$ $=A_{\text {teg }}-A_{\text {te }}$, and $T_{p v}^{4}-T_{a}^{4}=T_{h}^{4}-T_{c}^{4}$, and Eqs. (7) and (8) can be joined. In the present work all the above assumptions are taken, since they are well satisfied by the experimental setting and the system characteristics. Therefore, in the present case the effective device emittance is simply the average of the TEG and PV emittances $\left(\varepsilon_{e f f}=\frac{\varepsilon_{p v}+\varepsilon_{\text {teg }}}{2}\right)$ and radiative losses are calculated by Eq. (6).

It also useful to introduce the so-called TEG filling factor as

$F F=\frac{A_{t e}}{A_{\text {teg }}}$

Note that assumption 2 implies that the TEG filling factor is small. In view of assumption 1 , it follows then that $F F=\frac{A_{t e}}{A_{a b s}}$.

In Fig. 2 the typical behaviors of $\eta_{h t e p v}, \eta_{p v}, \eta_{t e g}$, and $\eta_{o t}$ as a function of $T_{h}$ are reported. It can be seen in the graph that while the PV and optothermal efficiencies decrease versus temperature, the TEG efficiency increases. This sets an optimal temperature of operation for the HTEPV system $\left(T_{\text {opt }}\right)$.

This is a key point for the engineering of the device. Actually, once the PV efficiency versus temperature and its optical characteristics $\left(R_{p v}\right.$, and $\varepsilon_{p v}$ ) are determined by characterization, and once also the TEG material properties versus temperature are defined, it is possible to predict the behavior of $\eta_{h t e p v}$ as a function of $T_{h}$ and determine $T_{\text {opt }}$. Based on the optimal working temperature the TEG leg sizes can be computed. In fact, the heat flowing through the TEG can be written as

$Q_{t e}=\left(\alpha_{p}-\alpha_{n}\right) T_{h} I+\frac{T_{h}-T_{c}}{R_{p}^{t h}+R_{n}^{t h}}-\frac{I^{2}\left(R_{p}^{e l}+R_{n}^{e l}\right)}{2}$

with $I$ the TEG electrical current, $R_{p}^{\text {th }}$ and $R_{n}^{\text {th }}$ the $\mathrm{p}$ and $\mathrm{n}$ thermoelectric materials thermal resistances, and $R_{p}^{e l}$ and $R_{n}^{e l}$ the $\mathrm{p}$ and $\mathrm{n}$ thermoelectric materials electrical resistances. The first, second, and third addend of Eq. (11) are respectively the Peltier heat, the conduction heat (Fourier's law), and Joule's heat.

The TEG leg sizes are represented into Eq. (11) by the following relationships:

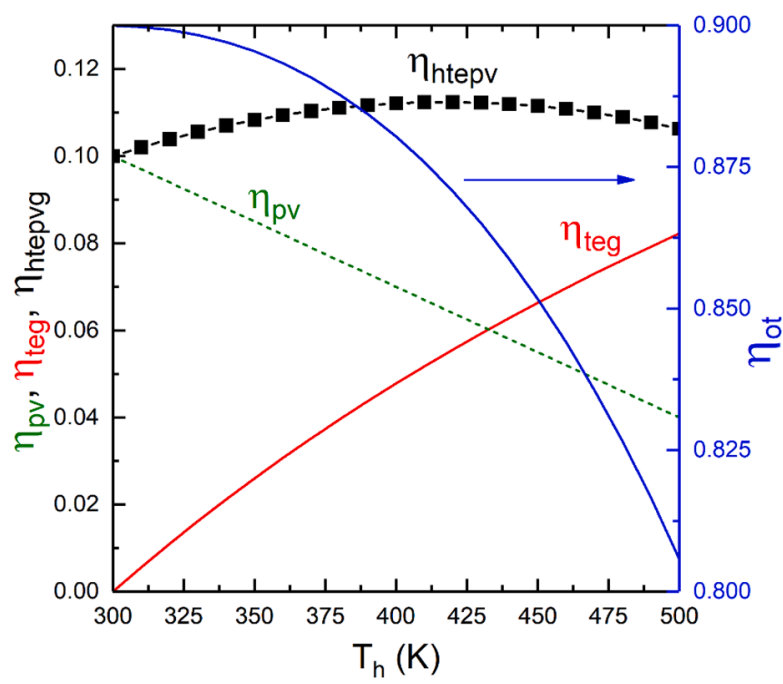

Fig. 2. Generic trends of $\eta_{h t e p v}, \eta_{p v}, \eta_{t e g}$, and $\eta_{o t}$ as a function of $T_{h}$. . Adapted from [39]
$R_{p}^{t h}=\frac{L_{p}}{\kappa_{p} A_{p}}, R_{n}^{t h}=\frac{L_{n}}{\kappa_{n} A_{n}}$

$R_{p}^{e l}=\frac{\rho_{p} L_{p}}{A_{p}}, R_{n}^{t h}=\frac{\rho_{n} L_{n}}{A_{n}}$

where $L_{p}, L_{n}$, and $A_{p}, A_{n}$ are respectively the $\mathrm{p}$ and $\mathrm{n}$ leg lengths, and cross-sectional areas. For the sake of simplicity in this work we will consider a TEG generator composed by only one p-n couple of thermoelectric materials, with the same lengths (namely $L_{p}=L_{n}=L$ ). It follows that $A_{t e}=A_{p}+A_{n}$.

Since the opto-thermal efficiency defines the efficiency with which the HTEPV system converts the incoming power into heat flowing through the TEG it follows that $Q_{t e}$ con be also be written as

$Q_{t e}=P_{i n} \eta_{o t}$

Therefore, once $T_{o p t}$ is defined, it is possible to compute $\eta_{o t}$ from Eq. (4), and then calculate the optimal leg dimensions using Eqs. (11), (12), (13), and (14) in order to keep the system working at optimal temperature. In the calculation it has to be taken into account that when the $p$ and $\mathrm{n}$ legs have the same thickness an optimal ratio between the $\mathrm{n}$ and $\mathrm{p}$ leg areas exists, and it is equal to

$\gamma_{n p}^{o p t}=\frac{A_{n}}{A_{p}}=\sqrt{\frac{\rho_{n} \kappa_{p}}{\rho_{p} \kappa_{n}}}$

Eq. (15) is valid not only for the HTEPV case, but it is a general argument firstly proposed by Ioffe [41] applicable to TEG generators when operating between two heat sinks.

Finally, it is useful to define the efficiency gain, hereafter named Energetic Convenience Index (EnCI), due to thermoelectric hybridization as

$E n C I=\eta_{h t e p v}-\eta_{p v}^{0}$

where $\eta_{p v}^{0}$ is the PV efficiency at room temperature. Thus, EnCI enables a quantitative comparison between HTEPV systems and the best PV scenario, namely cooled solar cells.

In summary, we firstly carried out a detailed characterization of three kind of wide-gap solar cells, in order to determine their efficiency dependence over temperature and their optical characteristics $\left(R_{p v}\right.$, and $\varepsilon_{p v}$ ). Then the results of this characterization are inputted along with the thermoelectric material properties in Eq. (1) in order to calculate the HTEPV efficiency behavior versus temperature and to find the optimal working temperature $T_{\text {opt }}$. Then using Eq. (14) the heat flowing through the TEG at $T_{\text {opt }}$ is calculated and using Eqs. (11), (12), (13), and (15), the thermoelectric leg areas $A_{n}$ and $A_{p}$ are found for a given value of the leg thickness $L$. Finally using Eq. (10) the value of FF can be determined.

\section{Results and discussion}

\subsection{Solar cell description}

In this work the solar cells chosen to be hybridized are based on aSi, InGaP, and Perovskite. aSi and InGaP solar cells were acquired respectively from the Italian National Energy Agency (ENEA) [42] and from the National Renewable Energy Laboratory (NREL) [43]. Perovskite solar cells (PSCs) with a direct (n-i-p) mesoporous structure, were instead developed at the University of Rome Tor Vergata.

The structure of mesoporous PSCs used in this work is displayed in Fig. 3(a). A compact- $\mathrm{TiO}_{2}$, acting as a blocking layer for hole injection into the FTO electrode was deposited via spray pyrolysis on a glass/FTO (Fluorinated Tin Oxide). The mesoporous- $\mathrm{TiO}_{2}$ layer, deposited via spin coating and sintered at $480{ }^{\circ} \mathrm{C}$, acts instead as scaffold layer for the perovskite growth. Both the compact- $\mathrm{TiO}_{2}$ and the mesoporous $\mathrm{TiO}_{2}$ form the Electron Transport Layer (ETL). A triple cation perovskite 
(a) PSC STRUCTURE

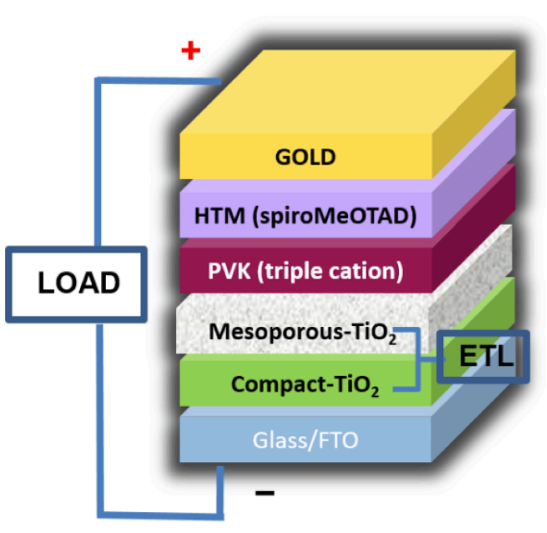

(b)

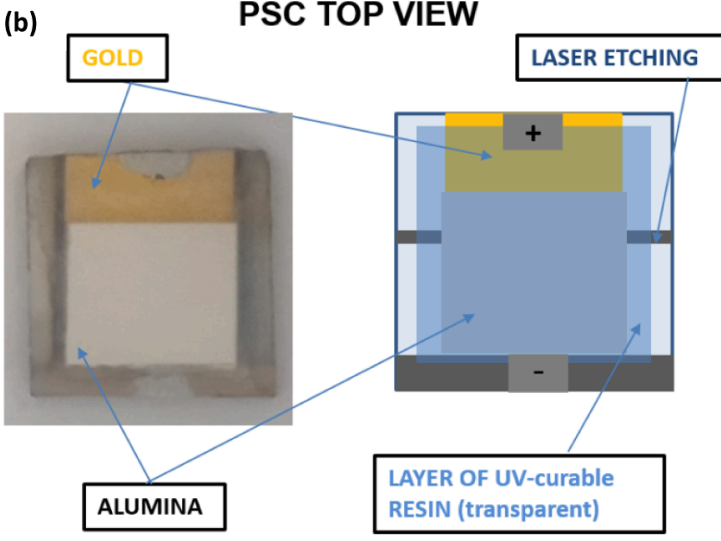

Fig. 3. Structure of the perovskite cells developed in this work. (a) Structure of our mesoporous PSC. From bottom to top: conductive glass substrate $(2.2 \mathrm{~mm})$ covered with a FTO layer (around $700 \mathrm{~nm}$ ), compact $\mathrm{TiO}_{2}$ layer (around $50 \mathrm{~nm}$ ), mesoporous $\mathrm{TiO}_{2}$ layer (around $200 \mathrm{~nm}$ ), perovskite layer (around $500 \mathrm{~nm}$ ), spiroMeOTAD layer (300 $\mathrm{nm}$ ), gold layer (around $100 \mathrm{~nm}$ ). (b) Top view of the PSC. A layer of UV-curable resin covers the area surrounding the alumina sheet that in turn covers the gold layer in correspondence of the active area of the PSC (located between the laser etching and the silver printed on the anode).
$\left(\mathrm{Cs}_{0.05} \mathrm{FA}_{0.8} \mathrm{MA}_{0.15} \mathrm{~Pb}\left(\mathrm{I}_{0.85} \mathrm{Br}_{0.15}\right)_{3}\right)$ absorber, with an energy gap around $1.65 \mathrm{eV}$ (see extrapolation of this value in the supplementary information and similar results in [44]) was deposited via spin coating over the mesoporous- $\mathrm{TiO}_{2}$ layer, using Chlorobenzene as antisolvent, with one step deposition technique [45]. Right after its deposition, perovskite was annealed at $100^{\circ} \mathrm{C}$, and a SpiroMeOTAD Hole Transport Material (HTM) was deposited via spin coating over it. Then, an Au back electrode was deposited by thermal deposition (i.e. vacuum evaporation at $10^{-6}$ bar). In this work, the glass substrate was $25 \times 25 \mathrm{~mm}^{2}$ and the FTO was etched ( $1 \mathrm{~mm}$ in width, see right) by means of a Nd:YVO $4, \lambda=1064 \mathrm{~nm}$ raster scanning laser, in order to separate the anode and the cathode.

The active area for the measurements of electrical parameters (PCE, $\mathrm{J}_{\mathrm{SC}}, \mathrm{FF}$ and $\mathrm{V}_{\mathrm{OC}}$ ) was $1 \mathrm{~cm}^{2}$. To protect the PSC from degradation due to oxygen and moisture [46], an encapsulation strategy similar to that reported by Matteocci et al. [47] has been used.

\subsection{Measurements and characterizations}

As reported in the previous section, the characterization needed to predict the HTEPV performances consists in the measurement of solar cells reflectance, emittances, and temperature sensitivity. Solar cell UV-vis reflectance was characterized between 250 and $2500 \mathrm{~nm}$ with a Jasco 570 spectrophotometer equipped with an integrating sphere. Emittance was instead estimated by infrared reflectance as

$\varepsilon(T)=\frac{\int B B(\lambda, T)[1-R(\lambda)-\tau(\lambda)] d \lambda}{\int B B(\lambda, T) d \lambda}$

with $B B(\lambda, T)$ the calculated spectral power density of a black body at

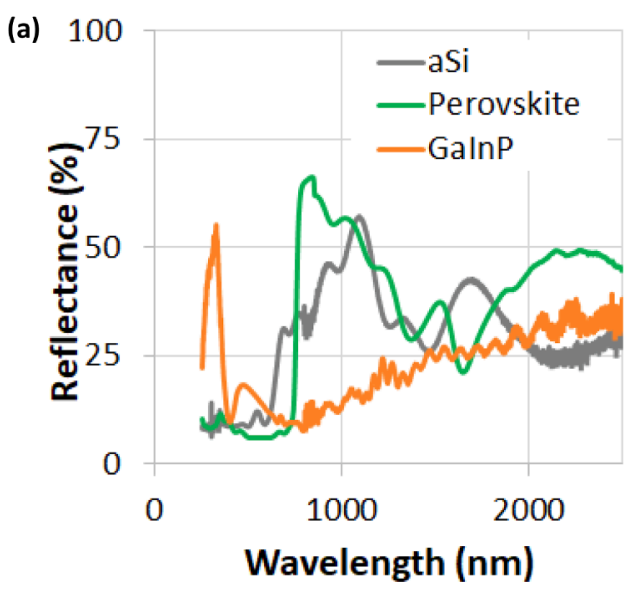

given temperature $T$ and wavelength $\lambda$, while $\tau(\lambda)$ and $R(\lambda)$ the measured transmittance and reflectance at the given wavelength. It has to be noted that for all the solar cells considered in this work $\tau(\lambda)=0$, since UV-Vis and IR transmittances were found to be null. Furthermore, in Eq. (17), $R$ depends only on $\lambda$ since the reflectance was taken at room temperature. Thus, in this evaluation we assumed a temperature independent reflectance. Fig. 4 shows emittance values for the three solar cells considered. Infrared reflectance was taken between 400 and $4000 \mathrm{~cm}^{-1}$ with a Thermo Fisher FTIR 6700 equipped with an integrating sphere.

Regarding current-voltage $(\mathrm{J}-\mathrm{V})$ characteristics of solar cells and of the hybrid device, the characterization was performed in a vacuum chamber equipped with a window and placed under an ABA, ABET Technologies solar simulator. For the solar cells, they were attached with thermally conductive paste on a controllable heather, and their temperature monitored with $\mathrm{K}$ type thermocouples and a Keithley 2700 multimeter. J-V curves were recorded between 25 and $80{ }^{\circ} \mathrm{C}$ with steps of $\sim 10{ }^{\circ} \mathrm{C}$ by a Keithley 2440 source meter controlled with a LabView program. Only for the case of perovskites, forward, reverse, and stabilized I-V curves have been recorded, since considerable differences were found among the three cases (details in SI). The characterization was performed for all three cells between 1 and 5 Suns to determine the effect of optical concentration on the temperature sensitivity. The solar simulator incoming power was always measured and adjusted with a certified refence silicon solar cell, and a $1 \mathrm{~cm}^{2}$ stainless-steel mask was implemented to accurately evaluate the incoming power density.

The results of the temperature sensitivity characterization performed on the solar cells at 1 sun are reported in Fig. 5. As the graph displays, while GaInP shows the expected linear decrease versus temperature,

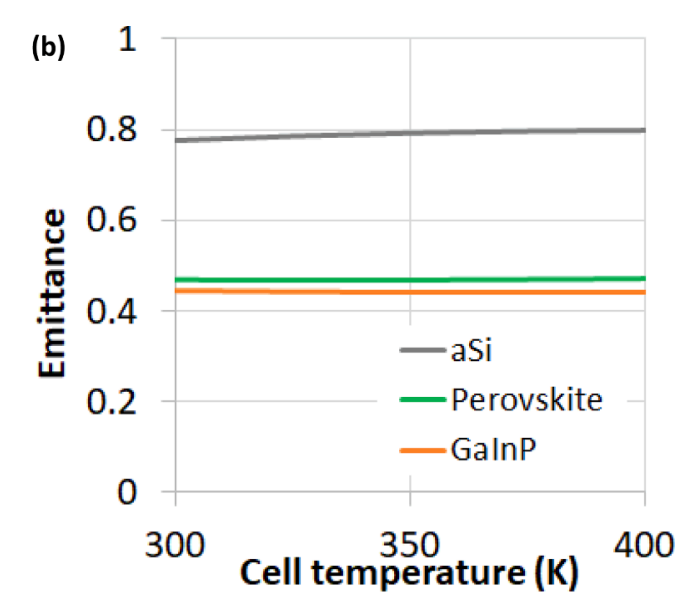

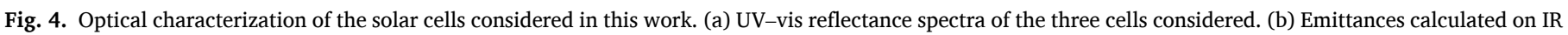
reflectance spectra of the three cells considered. 


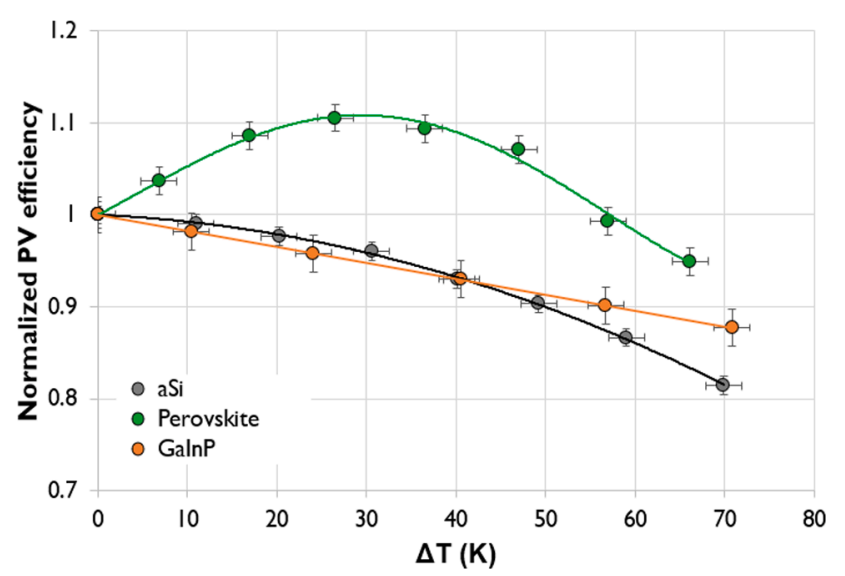

Fig. 5. Measured efficiency versus temperature at 1 Sun, normalized at their value at room temperature.

both aSi and perovskites exhibit a nonlinear behavior. Especially for the latter the efficiency shows an interesting increase, reaching a maximum in the temperature range $45-55^{\circ} \mathrm{C}$, followed by a strong decrease. This behavior is consistent with what reported in literature [48], and coherent with a tetragonal-to-cubic phase transition reported for MAPI perovskites [49-51]. Also regarding the cases of aSi [52] and GaInP [43], results are in line with literature data. For perovskites, Fig. 5 reports only the case of stabilized efficiencies, which are more reliable than the reverse and forward sweeps [53]. A detailed summary of the characterization on the perovskite and the other solar cells implemented in this work are reported in the Supplementary Information (SI). Finally, the temperature sensitivity of the three solar cells were fitted by polynomials (full lines in Fig. 5).

\subsection{Thermoelectric material}

The HTEPV system developed in this work is intended for low optical concentration levels. The reasons for this choice are several. First, low concentration implies optimal operation temperature typically under
$400 \mathrm{~K}$. Limited working temperatures are beneficial in terms of materials stability (especially for the solar cell), allow the use of only one thermoelectric material for the TEG part (avoiding the difficulties related to stacked or segmented TEGs), and keep the system in a temperature regime where other hybrid $\mathrm{PV} /$ thermal technologies are difficult to be implemented.

Secondly, at low concentration levels the system is more scalable, with limited occupational areas for the optics, with in addition the interesting possibility to avoid daily tracking (e.g. using Compound Parabolic Concentrators - CPCs). Finally, also regarding the dissipation at the TEG cold side, small concentration implies small heat flux to be dissipated and thus simplifying the system.

For temperatures between 300 and $400 \mathrm{~K}$ the most efficient and reliable thermoelectric material is bismuth telluride. For this reason, wafers of both $\mathrm{p}$ and $\mathrm{n}$ type $\mathrm{Bi}_{2} \mathrm{Te}_{3}, 1 \mathrm{~mm}$ thick, coated with titanium were acquired. Producer's data regarding the wafers thermoelectric properties are reported in the SI.

\subsection{Predicted efficiency gains}

Fig. 6 summarizes the results of the model based on the PV characterization described in the previous section. The graphs compare the efficiencies of the PV (dashed lines) and the HTEPV (full lines) as a function of temperature and optical concentration for the three PV materials considered in this paper. As anticipated, for all three cases, thermoelectric hybridization leads to efficiencies higher than the efficiency of the sole PV case at room temperature. These gains show partially similar trends among materials. In all cases, the higher the optical concentration, the higher the gain. This behavior is due to a double beneficial effect of concentration, which on one hand increases the PV efficiency and mitigates the PV temperature sensitivity, and on the other hand reduces the effect of radiative thermal losses. The first effect is due to a higher carrier generation/recombination ratio, which increases the PV efficiency and decreases its temperature sensitivity. The second effect is instead due to a higher opto-thermal efficiency. In fact, the increase of optical concentration increases the value of $P_{\text {in }}$ in Eq. (4), leaving instead $Q_{\text {rad }}$ unaltered.

For the cases of GaInP and aSi the optimal temperature increases
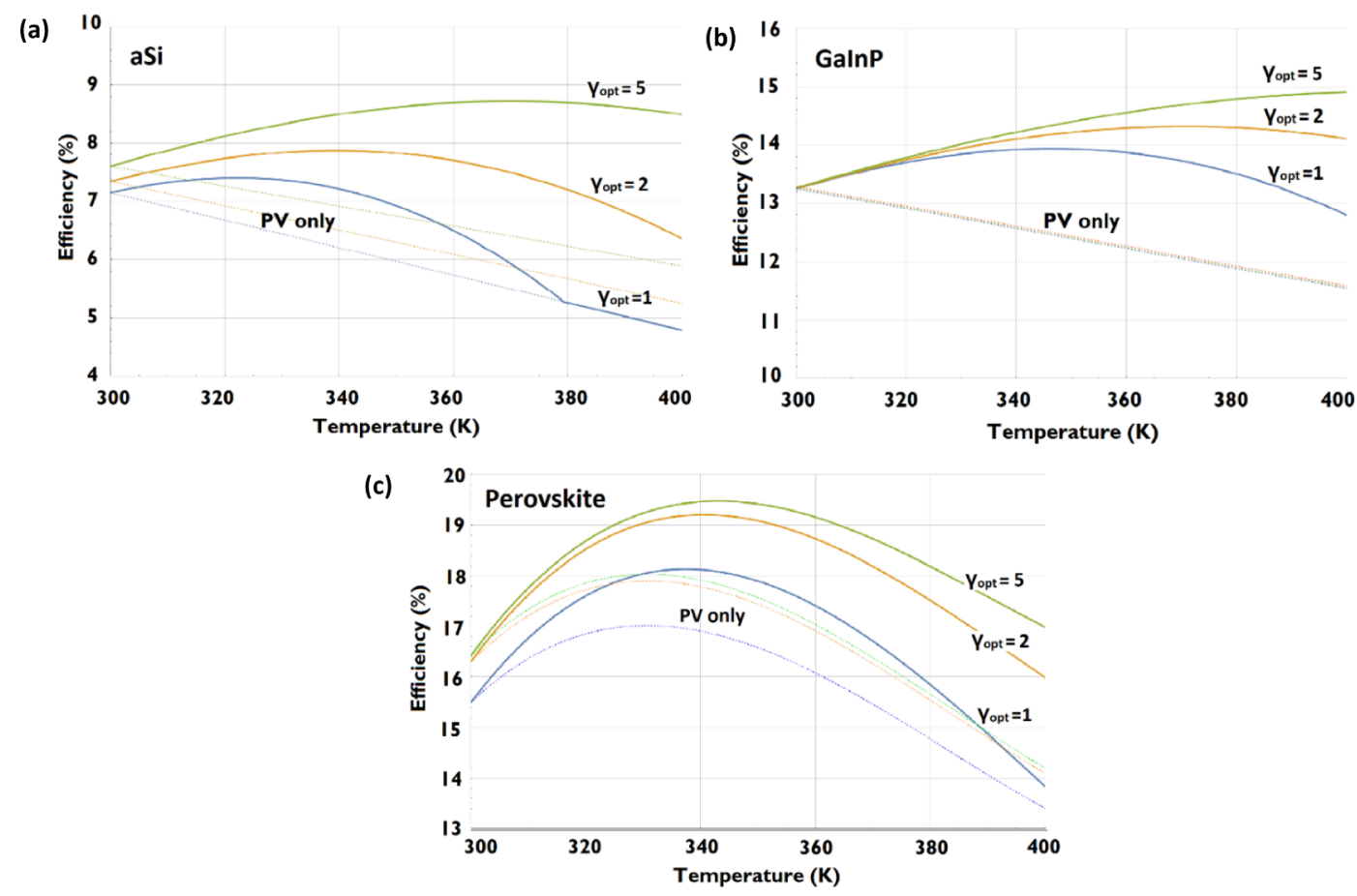

Fig. 6. Predicted $\eta_{p v}$ and $\eta_{h t e p v}$ as a function of temperature. (a) Amorphous silicon, (b) Gallium Indium Phosphide, (c) Perovskite. 
with the concentration. This implies that also the optimal TEG device (and thus the optimized output power) changes with the irradiation level. At concentration $5 \times$ the optical temperature falls in the range 370 - $400 \mathrm{~K}$. For perovskite, instead, the optimal temperature is stable around $340-350 \mathrm{~K}$ and is independent of the optical concentration. The main reason for this dissimilarity is given by the strong decrease of the perovskite PV efficiency for temperature higher than $340 \mathrm{~K}$.

Fig. 6 summarizes all efficiency gains (EnCI, as defined by Eq. (16)). In addition, for the case of perovskite, a further gain, defined as the difference between the maximum HTEPV efficiency and the maximum PV efficiency is reported, since in this case the PV efficiency at room temperature is not the PV best case scenario.

Table 1 clearly shows how perovskite gives the highest values of EnCI at all optical concentrations. This evidence, is further strengthened by an analysis of the economic viability of PSC-TE solar harvesters. As extensively discussed in a previous publication [54], hybridization of perovskite solar cells with TEGs at the current technology stage $(\mathrm{ZT} \approx 1)$ displays remarkable margins of profitability for domestic solar harvesting (rooftop solar panels). In short, we could show how, differently from what happens with more standard PV technologies (e.g. polycrystalline silicon), the additional capital cost of a TEG stage is largely overcompensated by the extra amount of electric power, both in nonconcentrated cells and for typical concentrations (5 suns) achievable with low-cost optical systems such as compound parabolic collectors. This, joined with the fact that perovskite has the higher final efficiency (as shown in Fig. 6) led to the choice of this material for the practical demonstration of solar cell hybridization.

\subsection{Optimized TEG development}

As mentioned above, in this work the hybrid system was implemented and fully validated for the case of perovskite. There are multiple reasons for this choice. Firstly, perovskite leads to the highest values of EnCI and of the final efficiency. Secondly, the model predicts an optimal temperature around $340 \mathrm{~K}$. This temperature is well within the range of temperatures normally experienced by solar panels and does not imply the need of difficult thermal management strategies. Thus, in this case the HTEPV device is directly comparable and compatible with actual solar cells. In addition the fact that the optimal temperature is essentially insensible to the variation of the irradiation level, also implies that the optimized TEG design is independent of this parameter. This is a very important evidence supporting the choice of perovskite solar cells, as it implies that the hybrid device will always work near its optimal temperature, independently of the change of the irradiation level over the day.

Using the model described in Sect. 2 the TEG filling factor and consequently the $\mathrm{p}$ and $\mathrm{n}$ leg areas were calculated. Table 2 reports the values of the filling factor for optical concentrations of 1,2 and 5 . As shown, filling factors are very small, justifying assumption 2 made in Sect. 2 .

\section{Table 1}

Values of EnCI for the three materials considered at $1 \times, 2 \times$, and $5 \times$ optical concentrations. Temperatures between rounds brackets report the temperature at which the maximum EnCI occurs.

\begin{tabular}{llll}
\hline & $\gamma_{\mathrm{opt}}=1$ & $\gamma_{\mathrm{opt}}=2$ & $\gamma_{\mathrm{opt}}=5$ \\
\hline $\mathrm{aSi}$ & $0.26 \%$ & $0.53 \%$ & $1.11 \%$ \\
& $(322.23$ & $(339.42$ & $(370.02$ \\
$\mathrm{GaInP}$ & $\mathrm{K})$ & $\mathrm{K})$ & $\mathrm{K})$ \\
& $0.68 \%$ & $1.07 \%$ & $1.64 \%$ \\
& $(346.47$ & $(370.86$ & $(408.29$ \\
Perovskite & $\mathrm{K})$ & $\mathrm{K})$ & $\mathrm{K})$ \\
& $2.64 \%$ & $2.90 \%$ & $3.05 \%$ \\
& $(337.43$ & $(340.59$ & $(343.13$ \\
Perovskite (gain with respect to the best & $\mathrm{K})$ & $\mathrm{K})$ & $\mathrm{K})$ \\
PV efficiency) & $1.12 \%$ & $1.30 \%$ & $1.44 \%$ \\
\hline
\end{tabular}

Table 2

Optimized TEG filling factor at different values of optical concentration.

\begin{tabular}{llll}
\hline & $\gamma_{\mathrm{opt}}=1$ & $\gamma_{\mathrm{opt}}=2$ & $\gamma_{\mathrm{opt}}=5$ \\
\hline Filling factor $\left(A_{t e} / A_{\text {abs }}\right)$ & $0.88 \times 10^{-2}$ & $1.95 \times 10^{-2}$ & $4.85 \times 10^{-2}$ \\
\hline
\end{tabular}

Based on the model results, $\mathrm{Bi}_{2} \mathrm{Te}_{3}$ wafers were then cut and soldered on copper plates (additional details in SI). Finally, the TEGs so obtained were characterized, measuring their internal resistance and their thermoelectric voltage. Fig. 7 left reports the I-V curve for the determination of the TEG internal resistance, while Fig. 7 right shows its thermoelectric response. For the sake of clarity Fig. 7 reports only the case of the TEG optimized to work at no optical concentration. The characterization performed showed that while Seebeck voltages were consistent with manufacturer's specifications, TEG internal resistance is slightly higher than the calculated value $(0.018 \Omega$ for the case reported in Fig. 7 left). This suboptimal result is presumably due to the existence of a contact resistance at the leg/plate interface, which in turn leads to a final HTEPV output power smaller than its calculated value.

\subsection{HTEPV characterization}

Once the TEG was characterized, the perovskite solar cell was thermally coupled to the TEG hot plate to form the HTEPV device. In particular, the TEG cold side was attached with thermal grease to the vacuum chamber bottom and its temperature controlled with a $\mathrm{K}$ type thermocouple. The chamber bottom temperature was controlled with a dissipation liquid circuit, fed with a chiller with adjustable temperature. In case of active cooling the temperature of the base was always kept at $25{ }^{\circ} \mathrm{C}$. This simulates a stable ambient temperature of $25{ }^{\circ} \mathrm{C}$, in the approximation that the water temperature equals that of the environment. In the real case scenario, however, the temperature of water fluctuates over time, moving the TEG cold temperature and in turns its output power. The solar cell was placed in thermal contact to the TEG top electrode by means of a layer of thermal grease. The grease was used to allow good thermal contact between the two components but also to allow the insertion of a thermocouple to measure the temperature at TEG/solar cell interface. Also in this case, J-V curves were recorded by a Keithley 2440 source meter controlled with a LabView program. The results reported in what follows were tested to be reproducible over several trials. Error bars shows the standard deviation of these measures.

Fig. 8(a)reports the influence of the active cooling on the build-up of a temperature difference at the TEG sides, in the case of no optical concentration. As expected, active cooling facilitates a more rapid increase of $\Delta \mathrm{T}$ over time and allows to reach a higher final temperature $(333.53 \mathrm{~K})$. This temperature is anyway slightly lower than the optimal temperature predicted by the model $(334.64 \mathrm{~K})$. The reason behind this discrepancy is probably due to the non-optimal thermal contact between the solar cell and the TEG hot plate. Actually, the model does not account for the thermal resistances of the alumina plate at the solar cell bottom and the thermal grease. Luckily, the predicted HTEPV efficiency (showed in Fig. 6) presents a region around the optimal temperature where the efficiency does not change significantly. This is a further strong point of HTEPV device based on perovskites. From Fig. 8(a) it might also seem that the passive and active cooling cases are not very different. However it should be considered that for the case of passive cooling the bottom of the vacuum chamber (consisting in a metallic plate with a large heat capacity due to its volume) acted as heat sink.

Fig. 8(b) reports instead the dependency of maximum $\Delta \mathrm{T}$ versus the chamber pressure. It shows that the maximum $\Delta \mathrm{T}$ is optimal for a range of pressure between $10^{-5}$ and $10^{-3}$ mBar but drops significantly for higher pressures. At ambient pressure the maximum $\Delta \mathrm{T}$ is nearly half of that at $10^{-5} \mathrm{mBar}$. Consequently, in this case the TEG contribution is a quarter of that at best case.

Finally, the output power of the TEG coupled with the perovskite 

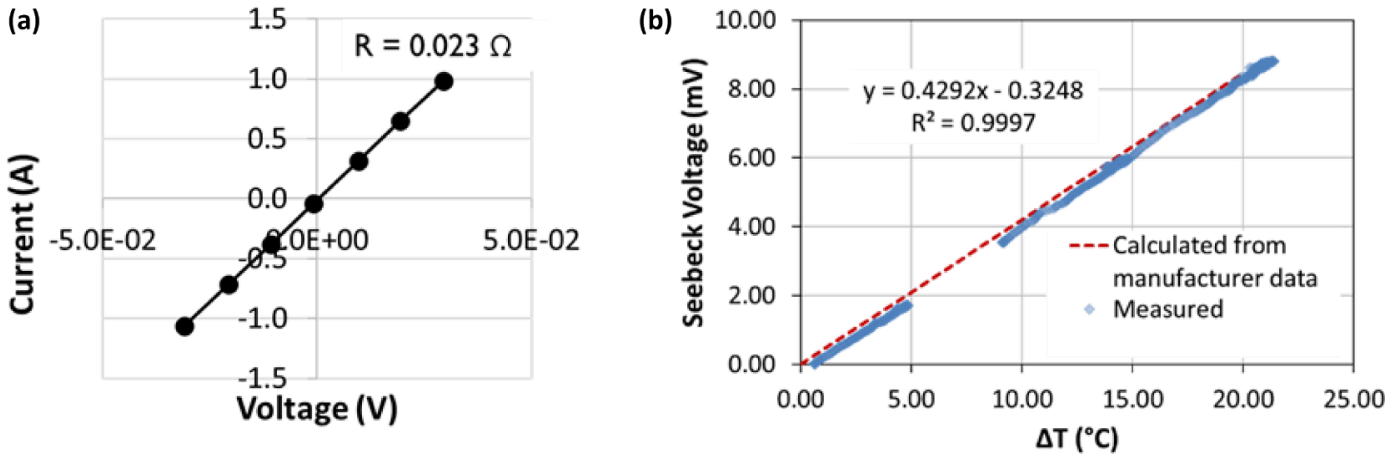

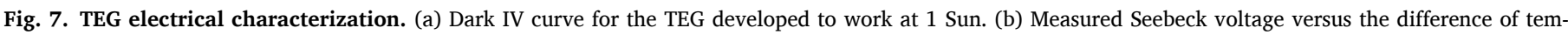
perature at the cold and hot plates of the TEG developed to work at 1 Sun, compared with the Seebeck voltage calculated on manufacturer's data.

(a) 40

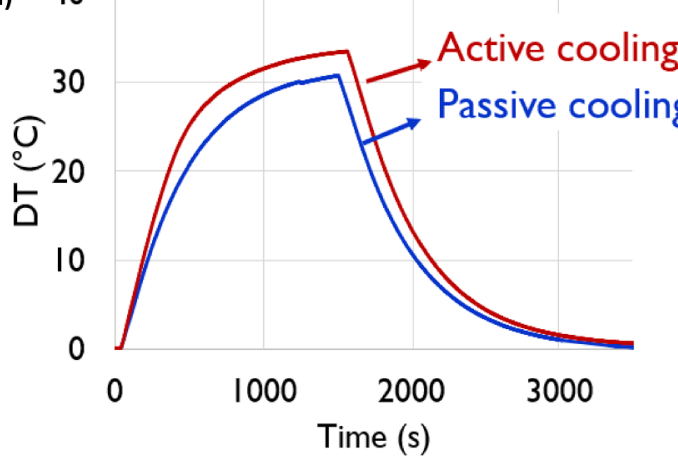

(b)

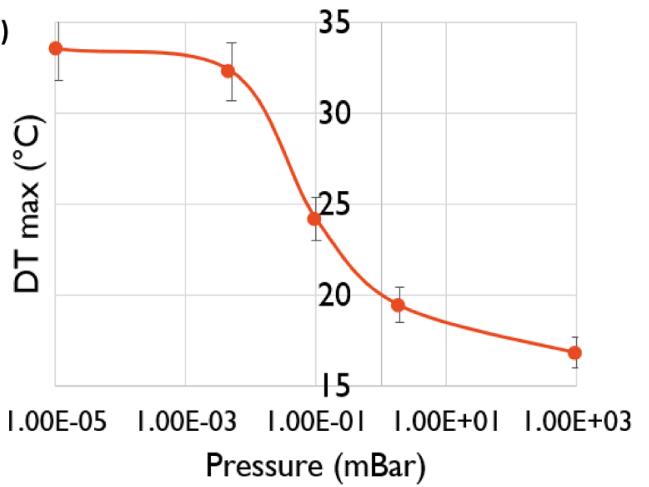

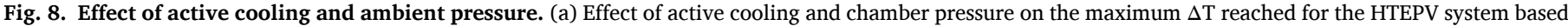
on perovskite at 1 Sun. (b) Effect of the chamber pressure.

solar cell under illumination at max $\Delta \mathrm{T}$ was measured by a $\mathrm{J}-\mathrm{V}$ characteristic. In Fig. 9 left the JV curve (black line) along with the power output, hereafter $P_{\text {teg }}$ (blue line) are reported. As expected the J-V characteristic is linear with an open circuit voltage $\left(\mathrm{V}_{\mathrm{oc}}\right)$ equal to the product between the TEG Seebeck coefficient and $\Delta \mathrm{T}$. Regarding the output power, the maximum TEG power, hereafter $P_{\text {teg }}^{\max }$ (which is the minimum of the blue line in Fig. 9(a), since by convention the output power of a power generator is negative) occurs at $\mathrm{V}_{\mathrm{oc}} / 2$ corresponding to a load resistance equal to the TEG internal resistance.

From the ratio $P_{\text {teg }}^{\max } / P_{\text {in }}$ it is then possible to calculate the TEG efficiency and consequently the value of the hybrid efficiency $\left(\eta_{h t e p v}\right)$ and of EnCI. Fig. 9(b) reports these results for three different value of optical concentrations compared with the model predictions, showing that the measured values of $\eta_{h t e p v}$ and EnCI follow quite accurately the predicted trend, although computations slightly overestimated them. This difference probably originates from a small mismatch of the TEG internal resistance, due to non-optimal electrical contacts between the legs and the copper plates.

Nevertheless, the comparison between the experimental characterization and the calculated data shows that our model is accurate.

\section{Conclusions}

In this work we demonstrated that the thermoelectric hybridization of wide-gap solar cells can lead to final efficiencies higher than the sole $\mathrm{PV}$ case. In particular the cases of aSi, GaInP and triple-cation perovskite
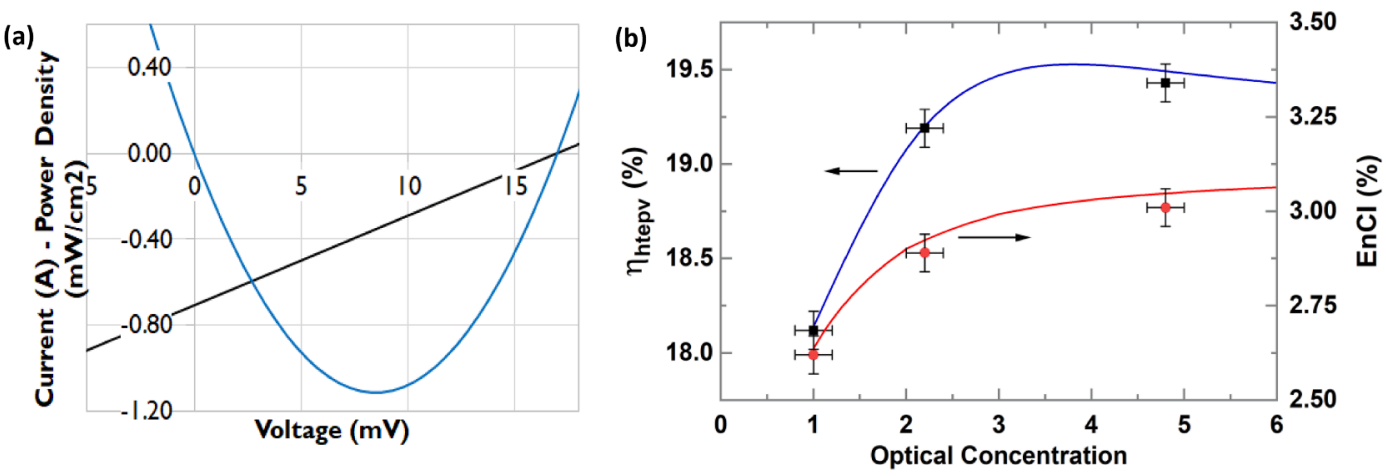

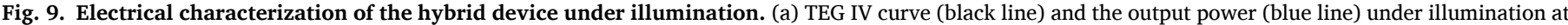

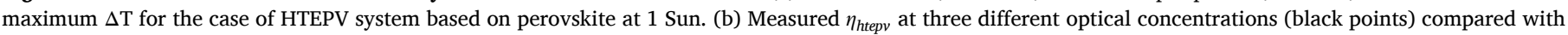

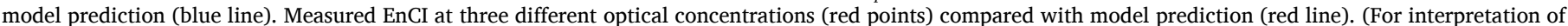
the references to colour in this figure legend, the reader is referred to the web version of this article.) 
were taken into account, and firstly analyzed in the framework of a computational model able to predict the final hybrid efficiency. The input data for the model were obtained by means of a detailed characterization of the given solar cells at different optical concentrations, predicting efficiency gains always positive, ranging between 0.2 and $3.1 \%$. The case of perovskites was found of particular interest since showed efficiency gains higher than $2 \%$ at all the optical concentrations considered (1, 2, and 5) with maximum efficiency gains happening at moderate temperatures (around $340 \mathrm{~K}$ ), nearly independent of the irradiation level. For these reasons and in view of the economic profitability of hybrid harvesters pairing TEGs and PSC cells, the case of perovskite was chosen for the development of a hybrid device prototype. To this purpose bismuth telluride wafers were acquired and cut in pieces accordingly to the sizes calculated with the model, able to make the system to work at optimal temperature. The thermoelectric legs were then soldered and the HTEPV system developed. The characterization of the system under solar illumination showed gains at optimal temperature very similar to those predicted, validating the model described and its results. The characterization also showed the influence of the pressure in which the system works, and the effect of active cooling at the TEG cold side. A final maximum efficiency gain of $3.05 \%$, at temperatures normally experienced by solar cells, was thus experimentally demonstrated for the case of perovskite, showing the actual potential of hybrid thermoelectric - photovoltaic systems.

\section{Declaration of Competing Interest}

The authors declare that they have no known competing financial interests or personal relationships that could have appeared to influence the work reported in this paper.

\section{Acknowledgements}

This project has received funding from the European Union's Horizon 2020 research and innovation program under the Marie Skłodowska-Curie Grant No. 745304.

\section{Appendix A. Supplementary data}

Supplementary data to this article can be found online at https://doi. org/10.1016/j.apenergy.2021.117343.

\section{References}

[1] Green MA. General temperature dependence of solar cell performance and implications for device modelling. Prog Photovoltaics Res Appl 2003;11:333-40. https://doi.org/10.1002/pip.496.

[2] Berthod C, Kristensen ST, Strandberg R, Odden JO, Nie S, Hameiri Z, et al. Temperature Sensitivity of Multicrystalline Silicon Solar Cells. IEEE J Photovoltaics 2019;9:927-64. https://doi.org/10.1109/JPHOTOV.2019.2911871.

[3] Peters IM, Liu H, Reindl T, Buonassisi T. Global Prediction of Photovoltaic Field Performance Differences Using Open-Source Satellite Data. Joule 2018;2:307-22. https://doi.org/10.1016/j.joule.2017.11.012.

[4] Polman A, Knight M, Garnett EC, Ehrler B, Sinke WC. Photovoltaic materials: Present efficiencies and future challenges. Science (80-) 2016;352.

[5] Shockley W, Queisser HJ. Detailed balance limit of efficiency of p-n junction solar cells. J Appl Phys 1961;32:422-7. https://doi.org/10.1063/1.1736034.

[6] Dupré O, Vaillon R, Green MA. A Thermal Model for the Design of Photovoltaic Devices. Therm. Behav. Photovolt. Devices Phys. Eng., Cham: Springer International Publishing; 2017. doi:10.1007/978-3-319-49457-9_3.

[7] Dupré O, Vaillon R, Green MA. Physics of the temperature coefficients of solar cells. Sol Energy Mater Sol Cells 2015;140:92-100. https://doi.org/10.1016/j. solmat.2015.03.025.

[8] Dupré O, Vaillon R, Green MA. A full thermal model for photovoltaic devices. Sol Energy 2016;140:73-82. https://doi.org/10.1016/j.solener.2016.10.033.

[9] Lorenzi B, Acciarri M, Narducci D. Experimental Determination of Power Losses and Heat Generation in Solar Cells for Photovoltaic-Thermal Applications. J Mater Eng Perform 2018;27:6291-8. https://doi.org/10.1007/s11665-018-3604-3.

[10] James A, Mohanraj M, Srinivas M, Jayaraj S. Thermal analysis of heat pump systems using photovoltaic-thermal collectors: a review 2021; vol. 144. https://doi. org/10.1007/s10973-020-09431-2.
[11] Shittu S, Li G, Akhlaghi YG, Ma X, Zhao X, Ayodele E. Advancements in thermoelectric generators for enhanced hybrid photovoltaic system performance. Renew Sustain Energy Rev 2019:24-54. https://doi.org/10.1016/j. rser.2019.04.023.

[12] Li G, Shittu S, Diallo TMO, Yu M, Zhao X, Ji J. A review of solar photovoltaicthermoelectric hybrid system for electricity generation. Energy 2018;158:41-58. https://doi.org/10.1016/j.energy.2018.06.021.

[13] Babu C, Ponnambalam P. The role of thermoelectric generators in the hybrid PV/T systems: A review. Energy Convers Manag 2017;151:368-85. https://doi.org/ 10.1016/j.enconman.2017.08.060.

[14] Xu L, Xiong Y, Mei A, Hu Y, Rong Y, Zhou Y, et al. Efficient Perovskite Photovoltaic-Thermoelectric Hybrid Device. Adv Energy Mater 2018:1702937.

[15] Liu Z, Sun B, Zhong Y, Liu X, Han J, Shi T, et al. Novel integration of carbon counter electrode based perovskite solar cell with thermoelectric generator for efficient solar energy conversion. Nano Energy 2017;38:457-66.

[16] Park K-T, Shin S-M, Tazebay AS, Um H-D, Jung J-Y, Jee S-W, et al. Lossless hybridization between photovoltaic and thermoelectric devices. Sci Rep 2013;3: 422-7.

[17] Zhang M, Miao L, Kang YP, Tanemura S, Fisher C a J, Xu G, et al. Efficient, low-cost solar thermoelectric cogenerators comprising evacuated tubular solar collectors and thermoelectric modules. Appl Energy 2013;109:51-9. doi:10.1016/j. apenergy.2013.03.008.

[18] Bjørk R, Nielsen KK. The performance of a combined solar photovoltaic (PV) and thermoelectric generator (TEG) system. Sol Energy 2015;120:187-94.

[19] Beeri O, Rotem O, Hazan E, Katz EA, Braun A. Gelbstein Y. Hybrid photovoltaic thermoelectric system for concentrated solar energy conversion: Experimental realization and modeling. J Appl Phys; 2015. p. 118.

[20] Mahmoudinezhad S, Ahmadi Atouei S, Cotfas PA, Cotfas DT, Rosendahl LA, Rezania A. Experimental and numerical study on the transient behavior of multijunction solar cell-thermoelectric generator hybrid system. Energy Convers Manag 2019;184:448-55. https://doi.org/10.1016/j.enconman.2019.01.081.

[21] Rajaee F, Rad MAV, Kasaeian A, Mahian O, Yan WM. Experimental analysis of a photovoltaic/thermoelectric generator using cobalt oxide nanofluid and phase change material heat sink. Energy Convers Manag 2020;212:112780. https://doi. org/10.1016/j.enconman.2020.112780.

[22] Yin E, Li Q, Li D, Xuan Y. Experimental investigation on effects of thermal resistances on a photovoltaic-thermoelectric system integrated with phase change materials. Energy 2019;169:172-85. https://doi.org/10.1016/j. energy.2018.12.035.

[23] Yin E, Li Q, Xuan Y. Optimal design method for concentrating photovoltaicthermoelectric hybrid system. Appl Energy 2018;226:320-9. https://doi.org/ 10.1016/j.apenergy.2018.05.127.

[24] Zhang J, Xuan Y. An integrated design of the photovoltaic-thermoelectric hybrid system. Sol Energy 2019;177:293-8. https://doi.org/10.1016/j. solener.2018.11.012.

[25] Zhou Y, Yin X, Zhang Q, Wang N, Yamamoto A, Koumoto K, et al. Perovskite solar cell-thermoelectric tandem system with a high efficiency of over $23 \%$. Mater Today Energy 2019;12:363-70. https://doi.org/10.1016/j.mtener.2019.03.003.

[26] Fu P, Qin W, Bai S, Yang D, Chen L, Guo X, et al. Integrating large-area perovskite solar module with thermoelectric generator for enhanced and stable power output. Nano Energy 2019;65:104009. https://doi.org/10.1016/j.nanoen.2019.104009.

[27] Chen G. Theoretical efficiency of solar thermoelectric energy generators. J Appl Phys 2011;109.

[28] Kraemer D, Poudel B, Feng H-P, Caylor JC, Yu B, Yan X, et al. High-performance flat-panel solar thermoelectric generators with high thermal concentration. Nat Mater 2011;10:422-7.

[29] Kraemer D, Jie Q, McEnaney K, Cao F, Liu W, Weinstein LA, et al. Concentrating solar thermoelectric generators with a peak efficiency of 7.4\%. Nat Energy $2016 ; 1$ : 16153.

[30] Narducci D, Lorenzi B. Challenges and Perspectives in Tandem ThermoelectricPhotovoltaic Solar Energy Conversion. IEEE Trans Nanotechnol 2016;15:348-55.

[31] Kraemer D, Hu L, Muto A, Chen X, Chen G, Chiesa M. Photovoltaic-thermoelectric hybrid systems: A general optimization methodology. Appl Phys Lett 2008;92. doi: 10.1063/1.2947591.

[32] Yang Z, Li W, Chen X, Su S, Lin G, Chen J. Maximum efficiency and parametric optimum selection of a concentrated solar spectrum splitting photovoltaic cellthermoelectric generator system. Energy Convers Manag 2018;174:65-71. https:// doi.org/10.1016/j.enconman.2018.08.038.

[33] Contento G, Lorenzi B, Rizzo A, Narducci D. Efficiency enhancement of a-Si and CZTS solar cells using different thermoelectric hybridization strategies. Energy 2017;131:230-8. https://doi.org/10.1016/j.energy.2017.05.028.

[34] Lorenzi B, Acciarri M, Narducci D. Conditions for beneficial coupling of thermoelectric and photovoltaic devices. J Mater Res 2015;30:2663-9. https://doi. org $/ 10.1557 / \mathrm{jmr} .2015 .174$.

[35] Gray JL. The physics of the solar cell. In: Steven H, editor. Antonio L. Handb. Photovolt. Sci. Eng.: John Wiley and Sons Ltd; 2003. p. 106-7.

[36] Lorenzi B, Acciarri M, Narducci D. Suitability of Electrical Coupling in Solar Cell Thermoelectric Hybridization. Designs 2018;2:32. https://doi.org/10.3390/ designs2030032.

[37] Singh P, Ravindra NM. Temperature dependence of solar cell performance-an analysis. Sol Energy Mater Sol Cells 2012;101:36-45. https://doi.org/10.1016/j. solmat.2012.02.019.

[38] Goldsmid HJ. Introduction to Thermoelectricity. vol. 121. Berlin, Heidelberg: Springer Berlin Heidelberg; 2010. doi:10.1007/978-3-642-00716-3. 
[39] Lorenzi B, Chen G. Theoretical efficiency of hybrid solar thermoelectricphotovoltaic generators. J Appl Phys 2018;124:024501. https://doi.org/10.1063/ 1.5022569 .

[40] Siegel R, Howell JR, Menguc MP. Thermal Radiation Heat Transfer. 5th ed. NY: Taylor \& Francis; 2002.

[41] Ioffe AF. Semiconductor thermoelements, and Thermoelectric cooling. Infosearch, 1td. 1957.

[42] Martella C, Chiappe D, Delli Veneri P, Mercaldo L V., Usatii I, Buatier De Mongeot F. Self-organized broadband light trapping in thin film amorphous silicon solar cells. Nanotechnology 2013;24:225201. doi:10.1088/0957-4484/24/22/225201.

[43] Perl EE, Simon J, Geisz JF, Lee ML, Friedman DJ, Steiner MA. Measurements and Modeling of III-V Solar Cells at High Temperatures up to $400{ }^{\circ} \mathrm{C}$. IEEE J Photovoltaics 2016;6:1345-52. https://doi.org/10.1109/ JPHOTOV.2016.2582398.

[44] Wang C, Zhang C, Wang S, Liu G, Xia H, Tong S, et al. Low-Temperature Processed, Efficient, and Highly Reproducible Cesium-Doped Triple Cation Perovskite Planar Heterojunction Solar Cells. Sol RRL 2018;2:1-8. https://doi.org/10.1002/ solr.201700209.

[45] Saliba M, Correa-Baena JP, Wolff CM, Stolterfoht M, Phung N, Albrecht S, et al. How to Make over 20\% Efficient Perovskite Solar Cells in Regular (n-i-p) and Inverted (p-i-n) Architectures. Chem Mater 2018;30:4193-201. https://doi.org/ 10.1021/acs.chemmater.8b00136.

[46] Shi L, Bucknall MP, Young TL, Zhang M, Hu L, Bing J, et al. Gas chromatographymass spectrometry analyses of encapsulated stable perovskite solar cells. Science (80-) 2020;368. doi:10.1126/science.aba2412.
[47] Matteocci F, Cinà L, Lamanna E, Cacovich S, Divitini G, Midgley PA, et al. Encapsulation for long-term stability enhancement of perovskite solar cells. Nano Energy 2016;30:162-72. https://doi.org/10.1016/j.nanoen.2016.09.041.

[48] Tress W, Domanski K, Carlsen B, Agarwalla A, Alharbi EA, Graetzel M, et al. Performance of perovskite solar cells under simulated temperature-illumination real-world operating conditions. Nat Energy 2019;4:568-74. https://doi.org/ 10.1038/s41560-019-0400-8.

[49] Cojocaru L, Uchida S, Sanehira Y, Gonzalez-Pedro V, Bisquert J, Nakazaki J, et al. Temperature effects on the photovoltaic performance of planar structure perovskite solar cells. Chem Lett 2015;44:1557-9. https://doi.org/10.1246/ cl.150781.

[50] Schelhas LT, Christians JA, Berry JJ, Toney MF, Tassone CJ, Luther JM, et al. Monitoring a Silent Phase Transition in CH3NH3PbI3 Solar Cells via Operando Xray Diffraction. ACS Energy Lett 2016;1:1007-12. https://doi.org/10.1021/ acsenergylett.6b00441.

[51] Garten LM, Moore DT, Nanayakkara SU, Dwaraknath S, Schulz P, Wands J, et al. The existence and impact of persistent ferroelectric domains in MAPbI 3. Sci Adv 2019;5:1-10. https://doi.org/10.1126/sciadv.aas9311.

[52] Virtuani A, Pavanello D, Friesen G. Overview of Temperature Coefficients of Different Thin Film Photovoltaic Technologies. Proc. 25th EU PVSEC 2010: 4248-52.

[53] Chen B, Yang M, Priya S, Zhu K. Origin of J-V Hysteresis in Perovskite Solar Cells. J Phys Chem Lett 2016;7:905-17. https://doi.org/10.1021/acs.jpclett.6b00215.

[54] Narducci D, Lorenzi B. Economic Convenience of Hybrid ThermoelectricPhotovoltaic Solar Harvesters. ACS Appl Energy Mater 2021;In press. doi:10.1021/ acsaem.1c00394. 